\title{
Evaluation of Variation Ranges of Phase Voltages and Ground Currents in Three-Phase Unearthed Networks With Diode Rectifiers
}

\author{
Piotr Olszowiec* \\ Erea Ltd.
}

\begin{abstract}
Operating parameters of electrical networks such as phase voltages, leakage and ground fault currents are decisive for safe operation of low-voltage unearthed networks with diode rectifiers. The knowledge of maximum possible values of network operating parameters is necessary for correct assessment of numerous hazards. So far no simple methods of determination of the highest levels of these parameters in AC/DC IT 3-phase networks have been available. The present paper reports a new approach to the evaluation of the variation ranges of the operating parameters of these systems using Mathcad.
\end{abstract}

Keywords - Diode rectifiers, ground-fault current, insulation resistance, leakage current, phase voltage, three-phase networks.

\section{INTRODUCTION}

Three-phase low voltage networks with unearthed neutral with rectifiers (AC/DC IT) (see Fig. 1) are commonly applied for the supply of auxiliary automation and control systems. An important task of the exploitation of these systems is maintaining it's insulation-to-ground at the required level. Network-to-ground insulation resistance exerts influence on such operating parameters as phase voltages, leakage, and ground-fault currents [1]. These parameters are decisive for a safe and correct operation of these networks. An excessive phase voltage rise (overvoltage) increases the risk of insulation breakdown, whereas high leakage and ground-fault currents may cause human shock, fire, or explosion [2].

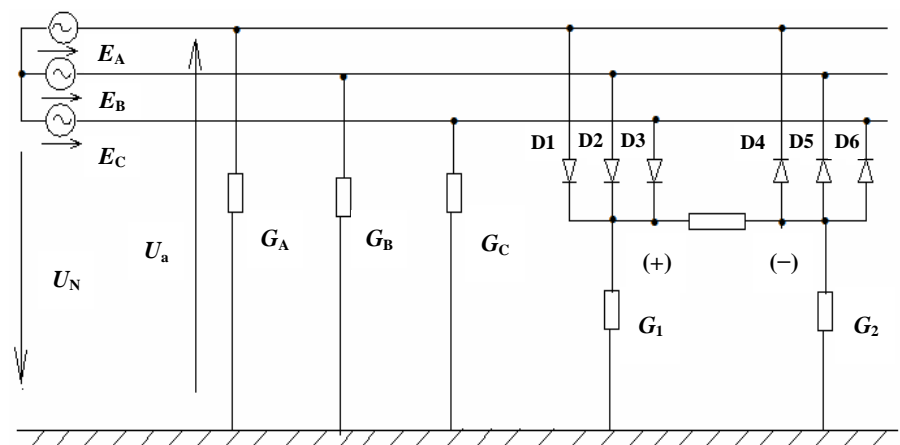

Fig. 1. Equivalent circuit of 3-phase IT network.

The knowledge of the maximum possible values of network operating parameters is necessary for correct assessment of the mentioned hazards. Some analysis of these parameters variation ranges in AC IT networks has been presented in [3]; however, no simple methods how to determine the highest levels of operating parameters in "mixed" (AC/DC) networks have been published so far.

\section{Network Neutral Point DisPlacement Voltage}

The network neutral (star) point displacement voltage $U_{\mathrm{N}}$ may be used as a starting point for determination of phase-toground voltages at $\mathrm{AC}$ side. Usually, network-to-ground capacitances can be neglected, which clearly simplifies analysis. This assumption has therefore been taken for further considerations. The time functions of symmetrical source phase voltages are as follows:

$$
\begin{aligned}
& e_{\mathrm{a}}(t)=E_{\mathrm{m}} \cdot \sin \omega t, e_{\mathrm{b}}(t)=E_{\mathrm{m}} \cdot \sin \left[\omega\left(t-\frac{T}{3}\right)\right], \\
& e_{\mathrm{c}}(t)=E_{\mathrm{m}} \cdot \sin \left[\omega\left(t-\frac{2 T}{3}\right)\right] .
\end{aligned}
$$

For convenience, the equivalent insulation conductances are designated at the AC side as $G_{i \mathrm{AC}}=G_{\mathrm{a}}+G_{\mathrm{b}}+G_{\mathrm{c}}$, and at the DC side as $G_{i \mathrm{DC}}=G_{1}+G_{2}$. In consecutive intervals of the rectifier diodes commutation, the momentary values of displacement voltage $u_{\mathrm{N}}(t)$ are given by the following formulas:

$T / 12-3 T / 12$ (D1, D5 diodes conduct):

$u_{N(1,3)}(t)=E_{m} \cdot \frac{\left(G_{a}+G_{1}\right) \cdot \sin (\omega t)+\left(G_{b}+G_{2}\right) \cdot \sin \left(\omega t-\frac{2 \pi}{3}\right)+G_{c} \cdot \sin \left(\omega t-\frac{4 \pi}{3}\right)}{G_{\text {idC }}+G_{\text {iDC }}}$

$3 T / 12-5 T / 12$ (D1, D6 diodes conduct):

$u_{N(3, s)}(t)=E_{m} \cdot \frac{\left(G_{a}+G_{1}\right) \cdot \sin (\omega t)+G_{b} \cdot \sin \left(\omega t-\frac{2 \pi}{3}\right)+\left(G_{c}+G_{2}\right) \cdot \sin \left(\omega t-\frac{4 \pi}{3}\right)}{G_{u C}+G_{i D C}}$

$5 T / 12-7 T / 12$ (D2, D6 diodes conduct ):

$u_{N(S, 7)}(t)=E_{m} \cdot \frac{G_{a} \cdot \sin (\omega t)+\left(G_{b}+G_{1}\right) \cdot \sin \left(\omega t-\frac{2 \pi}{3}\right)+\left(G_{c}+G_{2}\right) \cdot \sin \left(\omega t-\frac{4 \pi}{3}\right)}{G_{U C}+G_{D C C}}$

7T/12-9T/12 (D2, D4 diodes conduct):

$u_{N(T, 9)}(t)=E_{m} \cdot \frac{\left(G_{a}+G_{2}\right) \cdot \sin (\omega t)+\left(G_{b}+G_{1}\right) \cdot \sin \left(\omega t-\frac{2 \pi}{3}\right)+G_{c} \cdot \sin \left(\omega t-\frac{4 \pi}{3}\right)}{G_{i A C}+G_{i D C}}$

\footnotetext{
${ }^{*}$ Corresponding author.

E-mail address: olpio@o2.pl
} 
9T/12 - 11T/12 (D3, D4 diodes conduct):

$u_{N(9,11)}(t)=E_{m} \cdot \frac{\left(G_{a}+G_{2}\right) \cdot \sin (\omega t)+G_{b} \cdot \sin \left(\omega t-\frac{2 \pi}{3}\right)+\left(G_{c}+G_{1}\right) \cdot \sin \left(\omega t-\frac{4 \pi}{3}\right)}{G_{i A C}+G_{i D C}}$

11T/12 - 13T/12 (D3, D5 diodes conduct):

$$
u_{N(11,13)}(t)=E_{m} \cdot \frac{G_{a} \cdot \sin (\omega t)+\left(G_{b}+G_{2}\right) \cdot \sin \left(\omega t-\frac{2 \pi}{3}\right)+\left(G_{c}+G_{1}\right) \cdot \sin \left(\omega t-\frac{4 \pi}{3}\right)}{G_{i A C}+G_{i D C}}
$$

RMS value of displacement voltage is

$$
\begin{aligned}
& U_{\mathrm{Nrms}}=\operatorname{sqrt}\left[\frac{1}{T}\left(\int_{T / 12}^{3 T / 12} u_{\mathrm{N}(1,3)}^{2} d t+\int_{3 T / 12}^{5 T / 12} u_{\mathrm{N}(3,5)}^{2} d t+\int_{5 T / 12}^{7 T / 12} u_{\mathrm{N}(5,7)}^{2} d t\right)+\right. \\
& \left.+\frac{1}{T}\left(\int_{7 T / 12}^{9 T / 12} u_{\mathrm{N}(7,9)}^{2} d t+\int_{9 T / 12}^{11 T / 12} u_{\mathrm{N}(9,11)}^{2} d t+\int_{11 T / 12}^{13 T / 12} u_{\mathrm{N}(11,13)}^{2} d t\right)\right] .
\end{aligned}
$$

The analysis of dependence of this function on five independent variables, i.e., insulation conductances $G_{\mathrm{a}}, G_{\mathrm{b}}$, $G_{\mathrm{c}}, G_{1}$, and $G_{2}$ can be simplified by assuming fixed levels of equivalent insulation conductances separately for $\mathrm{AC}$ side $\left(G_{i \mathrm{AC}}\right)$ and for DC side $\left(G_{i \mathrm{DC}}\right)$. The exploration of the $U_{\mathrm{Nrms}}$ function shows that it assumes the maximum when the entire AC side insulation leakage conductance is lumped at one phase only, e.g., $G_{\mathrm{c}}=G_{i \mathrm{AC}}$; whereas the entire DC side insulation leakage conductance is lumped at one rectifier pole, e.g., $G_{1}=G_{i \mathrm{DC}}$. The $U_{\mathrm{Nrms}}$ function attains its minimum in case of uniform insulation leakage conductance distribution, i.e., $G_{\mathrm{a}}=G_{\mathrm{b}}=G_{\mathrm{c}}=G_{i \mathrm{AC}} / 3$ and $G_{1}=G_{2}=G_{i \mathrm{DC}} / 2$. It follows from the formulas (2)-(8) that both the maximal and minimal values of $U_{\mathrm{Nrms}}$ depend on the ratio $G_{i \mathrm{AC}} / G_{i \mathrm{DC}}$. The dependence of this voltage referred to RMS value of the source phase voltage $U_{\mathrm{Nrms}} / E_{\mathrm{rms}}$ on the $G_{i \mathrm{AC}} / G_{i \mathrm{DC}}=$ $R_{i \mathrm{DC}} / R_{i \mathrm{AC}}$ ratio, plotted in Fig. 2 , was determined with the help of the Mathcad program and confirmed at a laboratory model. As it is shown in this Figure, the highest value of displacement voltage is obtained for $R_{i \mathrm{DC}} / R_{i \mathrm{AC}}=0$ and equals $\sqrt{1+\frac{3 \sqrt{3}}{4 \pi}} \approx 1.19$.

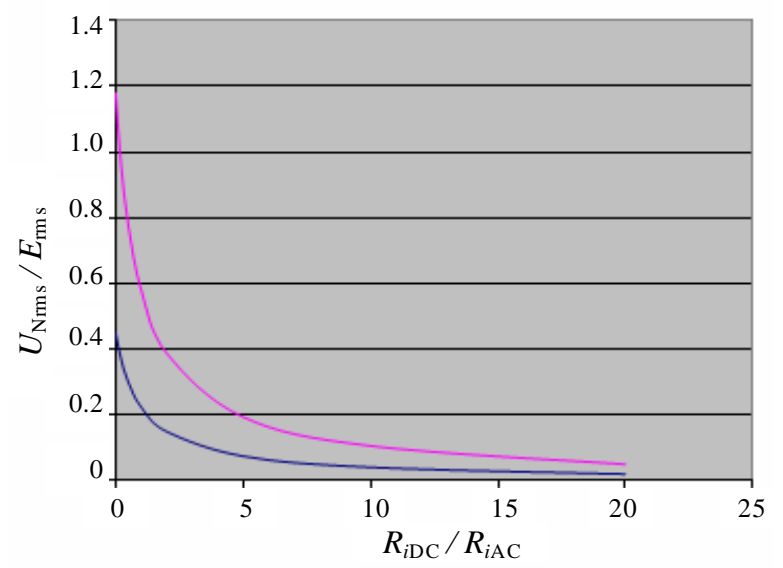

Fig. 2. Dependence of the maximum and minimum displacement voltage referred to the source phase voltage $U_{\mathrm{Nrms}} / E_{\mathrm{rms}}$ on the $R_{i \mathrm{DC}} / R_{i \mathrm{AC}}$ ratio.

From Fig. 2 it also follows that for any finite values of both equivalent insulation resistances $R_{i \mathrm{DC}}$ and $R_{i \mathrm{AC}}$, the displacement voltage is larger than zero. For comparison, in
AC IT networks (with no ground capacitances) without rectifiers: the phase voltages zero sequence value (displacement voltage) is obtained zero only for equal insulation resistances of all phases.

\section{Phase Voltages VARIATION RANGES}

For the analysis of phase (to ground) voltages variation, it is convenient to take advantage of the formulas (2)-(7) describing momentary values of displacement voltage $u_{\mathrm{N}}(t)$. The momentary values of phase voltage, e.g., "a", in consecutive intervals of the rectifier diodes commutation are equal to

$$
u_{\mathrm{a}(k, k+2)}=e_{\mathrm{a}}(t)-u_{\mathrm{N}(k, k+2)}(t), k=1,3,5,7,9,11 .
$$

Similarly to displacement voltage, the RMS value of phase voltage is

$$
\begin{aligned}
& U_{\mathrm{arms}}=\operatorname{sqrt}\left[\frac{1}{T}\left(\int_{T / 12}^{3 T / 12} u_{\mathrm{a}(1,3)}^{2} d t+\int_{3 T / 12}^{5 T / 12} u_{\mathrm{a}(3,5)}^{2} d t+\int_{5 T / 12}^{7 T / 12} u_{\mathrm{a}(5,7)}^{2} d t\right)+\right. \\
& \left.+\frac{1}{T}\left(\int_{7 T / 12}^{9 T / 12} u_{\mathrm{a}(7,9)}^{2} d t+\int_{9 T / 12}^{11 T / 12} u_{\mathrm{a}(9,11)}^{2} d t+\int_{11 T / 12}^{13 T / 12} u_{\mathrm{a}(1,113)}^{2} d t\right)\right] .
\end{aligned}
$$

As previously, it can be shown that RMS value of the phase "a" voltage assumes its maximum when the entire AC side insulation leakage conductance is lumped at one of the remaining phases, i.e., $G_{\mathrm{b}}=G_{i \mathrm{AC}}$ or $G_{\mathrm{c}}=G_{i \mathrm{AC}}$; whereas the entire DC side insulation leakage conductance is lumped at one rectifier pole. The function attains its minimum when $G_{\mathrm{a}}=G_{i \mathrm{AC}}$ and $G_{1}=G_{2}=G_{i \mathrm{DC}} / 2$. Just as in the case of displacement voltage, both maximal and minimal values of $U_{\mathrm{a} \text { rms }}$ depend on the ratio $G_{i \mathrm{AC}} / G_{i \mathrm{DC}}$. The dependence of this voltage referred to the RMS value of the source phase voltage $U_{\text {arms }} / E_{\mathrm{rms}}$ on $G_{i \mathrm{AC}} / G_{i \mathrm{DC}}=R_{i \mathrm{DC}} / R_{i \mathrm{AC}}$ is plotted in Fig. 3 . The highest value is obtained for $R_{i \mathrm{DC}} / R_{i \mathrm{AC}}=\infty$ and equals $\sqrt{3}$ (just as in the AC networks without ground capacitances).

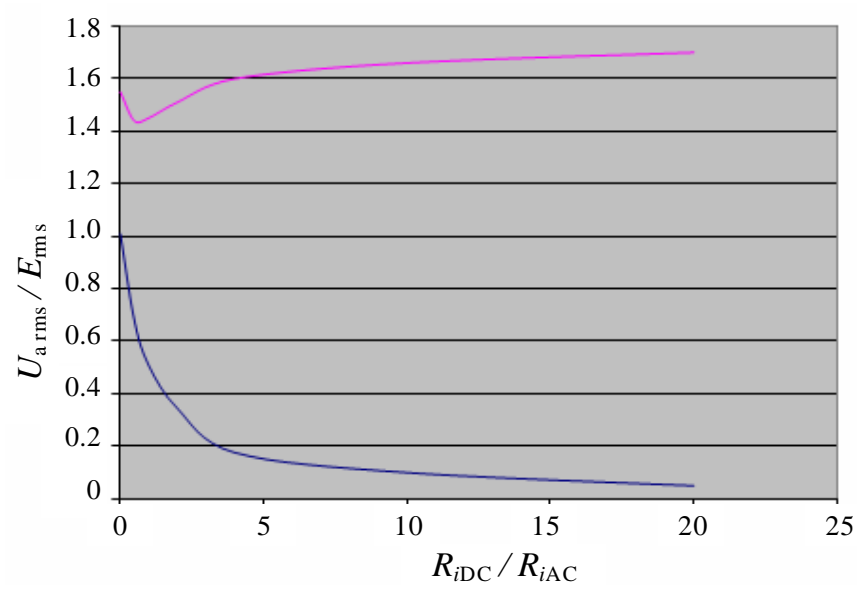

Fig. 3. Dependence of the maximum and minimum phase voltage referred to the source phase voltage $U_{\mathrm{a} \text { rms }} / E_{\mathrm{rms}}$ on the $R_{i \mathrm{DC}} / R_{i \mathrm{AC}}$ ratio. 
It follows from the Fig. 3 that in 3-phase networks with rectifiers, the variation range of phase voltage RMS values is somewhat smaller than for the networks without rectifiers. It should be remembered that in the AC IT system with the entire insulation conductance lumped in one phase only, its voltage is zero and the remaining phases voltages attain $1.73 \times$ rated level.

\section{DC COMPONENT OF PHASE VOLTAGES}

A characteristic feature of "mixed" networks is the presence of DC component in the AC side phase voltages, which is of course not possible in the AC IT systems [4]. This DC component (which is tantamount to non-zero mean value) appears when insulation leakage conductances of rectifier poles are not equal. In 3-phase AC/DC IT systems it is [1]:

$$
U_{\mathrm{a}-\text { mean }}=\frac{G_{1}-G_{1}}{G_{\mathrm{a}}+G_{\mathrm{b}}+G_{\mathrm{c}}+G_{1}+G_{2}} \cdot \frac{3 \sqrt{6}}{2 \pi} \cdot E_{\mathrm{rms}} .
$$

Just as in the case of displacement and phase RMS voltages, the highest mean value of the AC side voltage (which takes place for $G_{1}=G_{i \mathrm{DC}}$ or $G_{2}=G_{i \mathrm{DC}}$ ) also depends on the $R_{i \mathrm{DC}} / R_{i \mathrm{AC}}$ ratio (Fig. 4 ).

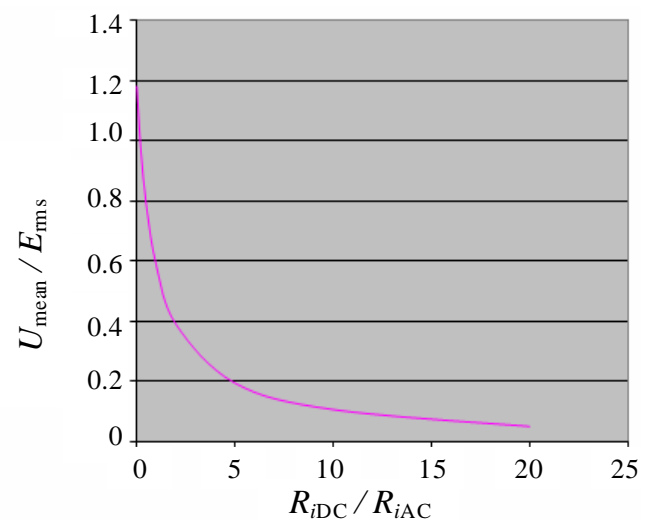

Fig. 4. Dependence of the maximum mean value of phase voltage referred to the source phase voltage $U_{\mathrm{a} \text { rms }} / E_{\mathrm{rms}}$ on the $R_{i \mathrm{DC}} / R_{i \mathrm{AC}}$ ratio.

The examples of the waveforms of the phase and displacement voltages in a 3-phase AC/DC network are shown in Fig. 5. These recordings confirm the possibility of a very high DC component content in the AC side voltages, which is the reason for distortion of these voltages.

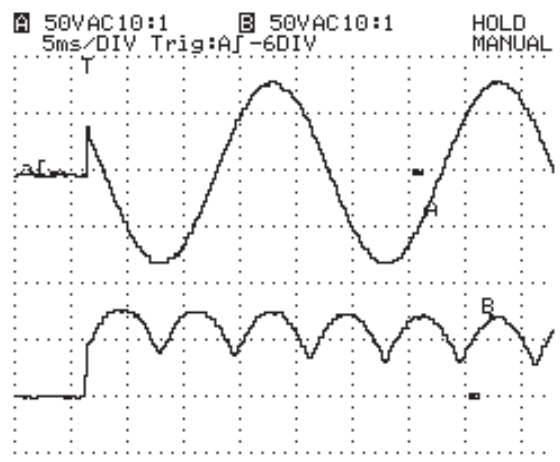

Fig. 5a. Waveforms of: A) source phase voltage $E_{\text {phrms }}=57 \mathrm{~V}$; B) displacement voltage in a network with insulation parameters $G_{\mathrm{a}}=G_{\mathrm{b}}=G_{\mathrm{c}}=G_{1 \mathrm{AC}}=0, G_{2}=G_{i \mathrm{DC}}\left(\right.$ i.e., $\left.R_{i \mathrm{DC}} / R_{i \mathrm{AC}}=0\right)$.

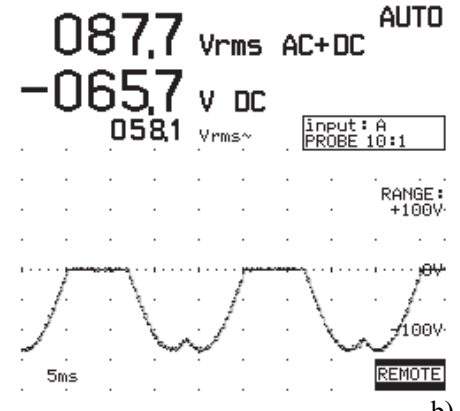

b)

Fig. 5b. Waveform of a phase voltage in the same network as in Fig. 5a.

In 3-phase IT networks, the displacement voltage is commonly used for detection of insulation-to-ground deterioration. In "mixed" networks, this criterion however cannot be used as neutral point voltage can assume considerable levels even with high insulation resistances (see the curve in Fig. 2) [5]. In fact, it may even reach the maximum value of $1.19 \times E_{\mathrm{ph} \text { rms, }}$ as in Fig. 5a. For comparison: in AC IT networks with equal phase-to-ground capacitances, the highest possible $U_{\mathrm{N}}$ value is $E_{\mathrm{ph}}$ in the case of a "dead" ground fault.

\section{LEAKAGE AND GROUND FAULT CURRENTS}

High leakage current, i.e., active component of the current flowing through insulation-to-ground conductances, poses threat of excessive temperature rise in places with a lowered insulation level. This may cause fire or explosion in the presence of flammable or explosive substances.

The leakage current (RMS value) in the phase, e.g., "a", is given by the following formula:

$$
I_{\text {a-leak }}=U_{\mathrm{a}-\text { rms }} \cdot G_{\mathrm{a}}
$$

As previously, it can be shown that RMS value of the phase "a" leakage current assumes its maximum when the entire AC side insulation leakage conductance is lumped at this phase, i.e., $G_{\mathrm{a}}=G_{i \mathrm{AC}}$, and the entire $\mathrm{DC}$ side insulation leakage conductance $G_{i \mathrm{DC}}$ is lumped at one of rectifier poles. The results of the maximum leakage currents measurements in a network with $E_{\mathrm{rms}}=1[\mathrm{~V}]$ are given in Table I.

\section{TABLE I}

MAXIMUM LEAKAGE CURRENTS $\left[\mu \mathrm{A}\right.$ ] IN A NeTWORK WITH $E_{\mathrm{RMS}}=1$ [V]

\begin{tabular}{|c|c|c|c|c|c|}
\hline$R_{i \mathrm{DC}}[\mathrm{k} \Omega]$ & 10 & 30 & 50 & 70 & 90 \\
\hline$R_{i \mathrm{AC}}[\mathrm{k} \Omega$ & & & & & \\
\hline 10 & 77 & 39 & 26.5 & 20.5 & 15.5 \\
\hline 30 & 39 & 26.5 & 19 & 16 & 13.5 \\
\hline 70 & 25 & 20 & 16 & 14 & 11 \\
\hline 90 & 19 & 17 & 13.5 & 12 & 10 \\
\hline
\end{tabular}

The surface plotted in Fig. 6 according to the formulas (2)(7), (9), (10), and (12) presents dependence of the maximum leakage current $I_{\mathrm{a}} \max$ RMS value on insulation equivalent resistances. Fig. 6 (and Table I with the help of linear interpolation) can be used for an approximate, quick evaluation of the sought maximum level of the leakage current at the AC side. It is worth to note [2] that in LV networks, dissipation of a $60 \mathrm{~W}$ thermal power in a limited part of insulation poses a threat of impermissible temperature rise 
leading to inflammation. For phase voltage rated level of $230 \mathrm{~V}$, this power is achieved with the leakage current of about $0.23 \mathrm{~A}$.

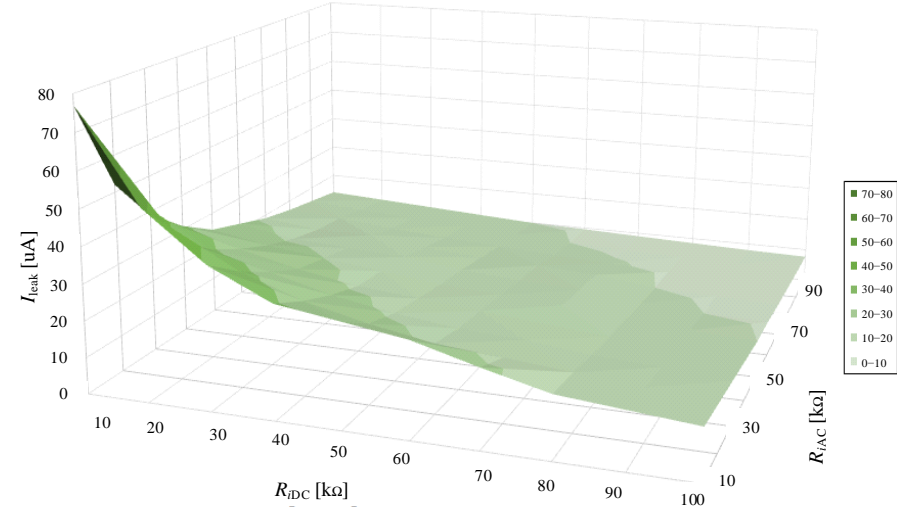

Fig. 6. Dependence of the AC side maximum leakage current (RMS value) for source phase voltage $E_{\mathrm{rms}}=1[\mathrm{~V}]$ on $R_{i \mathrm{DC}}$ and $R_{i \mathrm{AC}}$.

In a similar way, the maximum ground fault current at the AC side is evaluated. Also in this case, the phase-to-ground current assumes its maximum when the entire DC side insulation leakage conductance is lumped at one of rectifier poles.

The results of the maximum short circuit current measurements in a network with $E_{\mathrm{rms}}=1$ [V] are given in Table II. In Fig. 7, the dependence of the AC side maximum "dead" ground fault current on $R_{i \mathrm{DC}}$ and $R_{i \mathrm{AC}}$ is presented.

TABLE II

MAXIMUM SHORT CiRCUIT CURRENTS [ $\mu \mathrm{A}$ ] IN A NETWORK WiTH $E_{\text {RMS }}=1$ [V]

\begin{tabular}{|c|c|c|c|c|c|}
\hline$R_{i \mathrm{ACC}}[\mathrm{k} \Omega$ & 10 & 30 & 50 & 70 & 90 \\
\hline 10 & & & & & \\
\hline 30 & 190 & 210 & 193 & 187 & 183 \\
\hline 50 & 177 & 102 & 80 & 72 & 69 \\
\hline 70 & 169 & 74 & 59 & 51 & 48 \\
\hline 90 & 165 & 70 & 46 & 42 & 38 \\
\hline
\end{tabular}

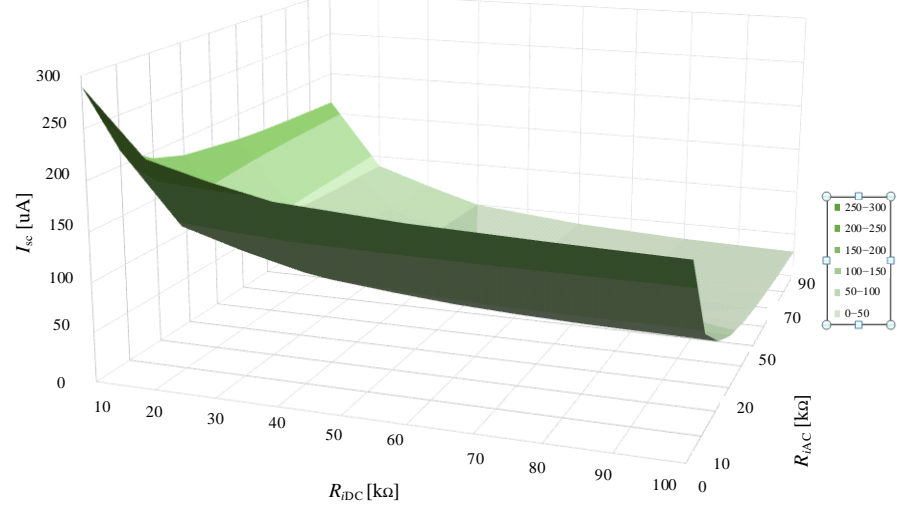

Fig. 7. Dependence of the maximum AC side "dead" phase-to-ground fault current (RMS value) for the source phase voltage $E_{\mathrm{rms}}=1$ [V] on $R_{i \mathrm{DC}}$ and $R_{i \mathrm{AC}}$.
It is worth to note that - similarly to the phase and displacement voltages - the AC side leakage and ground fault currents contain not only alternating (harmonics of all rows) but also DC component. The value of this component (i.e., mean value) of leakage current plays an important role among other components in electrochemical corrosive processes. This value in the phase, e.g., "a", is calculated as

$$
I_{\text {a-mean -leak }}=U_{\text {a-mean }} \cdot G_{\text {a }}
$$

It can be checked that the highest share of DC component in the "dead" ground fault current (RMS value) is approximately $53 \%$.

\section{Practical Utilization of Research Results}

The drawings shown in Figs 2-7 contain the results of research on the variation of voltages and currents at the $\mathrm{AC}$ side of 3-phase "mixed" networks. These data can be easily utilized in practice. As an example, let us evaluate the maximum values of the phase and displacement voltages as well as the leakage and "dead" ground fault currents in a $3 \times 400 \mathrm{~V}$ rated voltage network with insulation equivalent resistances $R_{i \mathrm{DC}}=R_{i \mathrm{AC}}=50 \mathrm{k} \Omega$. Any distribution of insulation leakage resistances between single phases and rectifier poles should be assumed as possible. In this network, $E_{\mathrm{rms}}=400 / 1.73=231 \mathrm{~V}$.

For $R_{i \mathrm{DC}} / R_{i \mathrm{AC}}=1$ and $E_{\mathrm{rms}}=1 \mathrm{~V}$, we read the maximum values of $U_{\mathrm{N}}=0.77 \mathrm{~V}$ and $U_{\mathrm{ph}}=1.45 \mathrm{~V}$ in Fig. 3 and Fig. 4, respectively. In Table $\mathrm{I}$, we read the maximum value of $I_{\text {leak }}=0.018 \mathrm{~mA}$, and in Table II $-I_{\mathrm{sc}}=0.058 \mathrm{~mA}$. For $E_{\mathrm{rms}}=231 \mathrm{~V}$, we obtain the maximum values of $U_{\mathrm{N}}=0.77 \times$ $231=178 \mathrm{~V}, \quad U_{\mathrm{ph}}=1.45 \times 231=335 \mathrm{~V}, I_{\text {leak }}=0.018 \times$ $231=4.2 \mathrm{~mA}$, and $I_{\mathrm{sc}}=0.058 \times 231=13.4 \mathrm{~mA}$. According to formula (11), the highest mean value of the phase voltage is $135 \mathrm{~V}$.

\section{CONCLUSION}

1. Three-phase unearthed networks with diode rectifiers exhibit some distinctive differences as compared to the $\mathrm{AC}$ IT systems, first of all, possibility of the mean value of high-phase voltages.

2. The maximum levels of the phase and displacement voltages are obtained when the entire $\mathrm{AC}$ side insulation leakage conductance is lumped at one phase only, whereas the entire DC side insulation leakage conductance is lumped at one of rectifier poles.

3. In AC/DC IT networks, the highest possible level of phase voltages is the same as in AC IT systems (with negligible ground capacitances) and equals $1.73 \times$ rated phase voltage.

4. Due to the DC component presence, displacement voltage may reach the highest level of $1.19 \times$ rated phase voltage.

5. The maximum leakage and ground fault currents can be determined with the help of enclosed tables (measurement results) and diagrams (calculation results). 


\section{REFERENCES}

[1] P. Olszowiec, Insulation Measurement and Supervision in Live AC and DC Unearthed Systems, Springer, 2012, vol. 167, pp. 5-15, ISBN 978-3-642-29754-0.

[2] W. Hofheinz, Protective Measures with Insulation Monitoring, VDE Verlag, 2006, pp. 63-72.

[3] C. Olszowiec and P. Olszowiec, "Evaluation of Variation Ranges of Electrical Parameters in Three-Phase Unearthed Networks," Computational Problems of Electrical Eng., 2014, vol. 4, no.1, pp. 51-54.

[4] E. Ivanov, "Kak prawilno izmieriat soprotivlenije izolacji elektroustanovok," Novosti Elektrotechniki, no. 2, 2002. [Online]. Available: http://www.news.elteh.ru/arh/2002/14/26.php (in Russian)

[5] P. Olszowiec, "Application of Network Voltages for Insulation Monitoring in Unearthed AC Circuits with Rectifiers," Pomiary, Automatyka, Kontrola, no. 12, pp. 1252-1255, 2013. 